\title{
Tales from the $C$. difficile trenches
}

Admitted to hospital for pneumonia, the retired man was visited every day by his wife, who helped feed and care for him. Then he contracted Clostridium difficile from a roommate and had to be transferred to intensive care. Next, his wife was admitted to another part of the hospital - with $\mathrm{C}$. difficile. She was too ill to visit him as he lay dying and too sick to attend his funeral.

$\mathrm{A}$ true story, one that Dr. Michael Gardam tells to bring home the human toll of an infection that is so often transmitted in hospital. "Things like this happen in hospitals and telling them is how we recognize the impact that hospitalacquired infections can have on patients," explains the director of infectious disease prevention and control at the Ontario Agency for Health Protection and Promotion.

The case also helped to spur change at the Toronto Western Hospital, says Teri Arany, nurse manager for a generally internal medicine ward.

In other instances, change has been more systemic, says Dr. John Wright, who was appointed president of the Scarborough General Hospital in 2008. The General was prompted to act after the province of Ontario began public reporting of hospital $C$. difficile rates and the institution had the dubious distinction of topping the list.

Edited transcripts of conversations with Arany and Wright about their experiences with $C$. difficile are presented below.

\section{Teri Arany, nurse manager, Toronto Western Hospital:}

Dispelling myths was one key to changing practice.

About a year and a half ago, a couple of serious $C$. difficile outbreaks at our hospital led to units being closed, admissions cancelled and restrictions placed on visitors. For a while, it was like a war zone of closed areas, and we realized this has to stop.

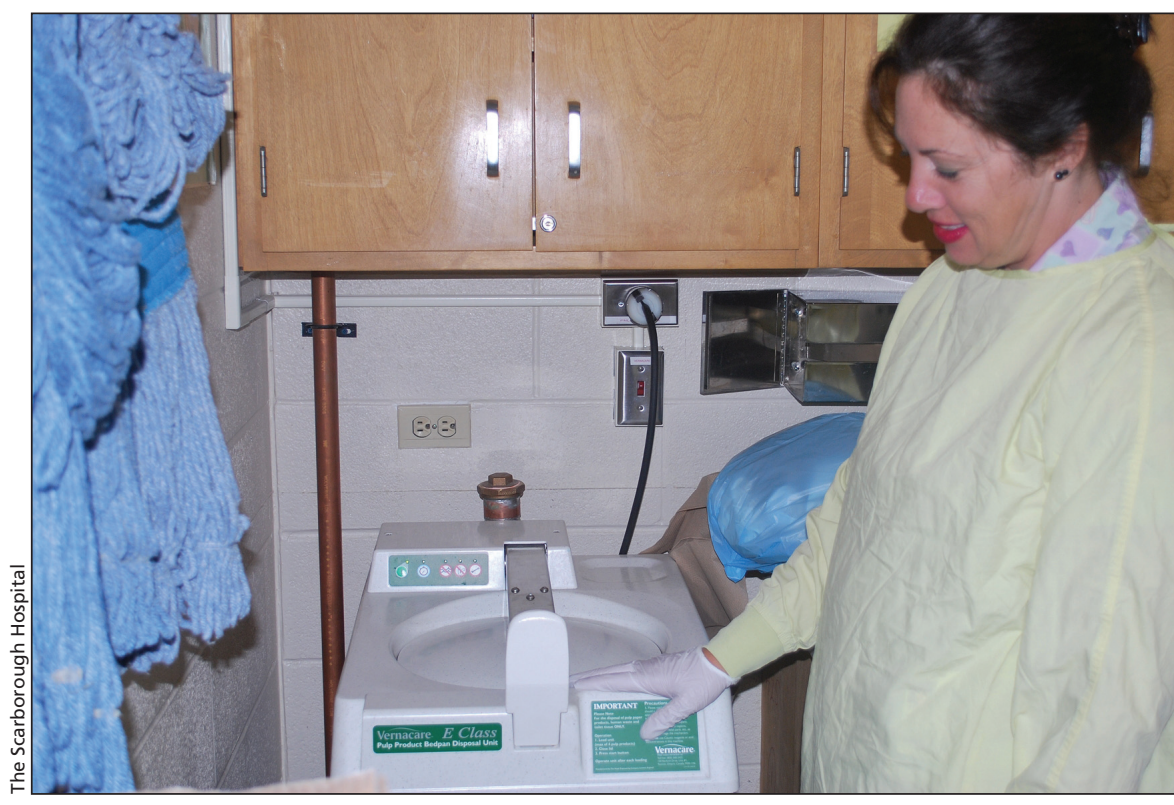

Registered practical nurse Connie Storr at a Vernacare machine, a disposal unit for fibre-disposable receptacles and maceratable wipes.

There had been initiatives to bring down rates in the past but early successes later fell apart. Part of the problem was that because rates were reported by units, they were being blamed, along with the nurses who worked within the units. We realized we needed to think broadly and create a strategic plan. We had to trust the expertise of units and give them the resources and time to create a sustainable practice. In the past, infection prevention and control had always been viewed as someone else's problem - this is just one of the challenges that we had.

When a hand-hygiene pilot project was launched, staff expressed the belief that hand gel did not do anything. So we had to have a huge cultural shift to an acceptance of hand gel as an adequate, if not superior, method. We spent lots of time on this.

Whenever we ran into cultural myths like this, we brought in experts and held town halls. Some staff argued that they had a dermatological reaction and therefore couldn't wash their hands. I removed them from patient care. I said, if you work on this unit, you have to be able to wash your hands. I sent them for a dermatological consult.

One person did have a bona fide reaction, so we worked with a dermatologist to find an appropriate handcleaning solution.

We made expectations very clear, referred to professional guidelines and had one-to-one chats with every staff member. A lot of the difficult work was soft work.

Another lesson was around the proper wearing of protective equipment and clothing. Often nursing and housekeeping staff were not comfortable engaging with other members of the team so we spelled out expectations, wrote them down and then helped them by role playing how they should approach doctors who were not using correct practice. We provided training to the physician residents who came through every two months, while our patient education department instructed visiting family members about our protocols.

We also decided that any piece of medical equipment should not touch 
more than two patients, so we got rid of the fancy $\$ 6000$ automatic blood pressure cuffs and instead fixed in place a manual cuff and thermometer at the head of every bed.

We used to have different housekeepers at different times. We decided we need significantly more staff and more staff who were dedicated to the ward. Organizationally, it costs a heck of a lot more for a nosocomial length of stay with medical care than to have extra cleaners.

Now, we maintain a big calendar and we count nosocomial free days and we have 90-day parties. Our last big outbreak was January 2007. Before our last outbreak, nursing staff had not had the best relationship with infection prevention control because infection control staff came, made recommendations and left. So nurses felt they weren't involved. Now, there is a partnership model. Precautionary measures are introduced at the first sign of a loose stool, for example. A sample is taken, the doctor is told and IPAC (infection prevention and control) is notified. Patients are cleared by infection control staff, not by doctors. Now, our whole point of view is that each area has to own the issue of preventing hospital-acquired infections.

\section{Dr. John Wright, president, Scar- borough General Hospital:}

Just because a hospital is old doesn't mean rates can not be drastically improved. Tossing out old fabric furniture and increasing and educating cleaning staff were important steps.

When the province first began public reporting of $C$. difficile in the fall of
2008, our facility had 17 cases of the hospital-acquired infection. Now we are down to one or two a month.

Reporting is important, certainly, because what gets measured gets attended to.

The General was built like topsy in 1956. It has big rooms, not enough washrooms and is difficult to clean. Older facilities pose big problems and this is the excuse that we all used for living with high rates of $C$. difficile, but we can't just put our hands in our pockets and walk away.

Within the hospital, medical wards classically have the highest rates of $C$. difficile because of the number of elderly patients with multisystem diseases, many of whom are on antibiotics and in and out of hospital. As a gastroenterologist, I was troubled. When I went to the wards, there were vast numbers in isolation with $C$. difficile, which is really expensive to look after, since patients have complications, need private rooms and stay longer in hospital.

We brought in a consultant who recommended changing cleaning techniques. We took the advice but then nothing happened. It turned out that although we had the proper recommended cleaning solution for rescue cleaning, Virox 5, frontline housekeepers thought it was dangerous and weren't using it.

In the end, it was just education that prompted the housekeepers to accept and use the disinfectant. We hired 18 temporary cleaners to do a surge cleaning. We also made a concerted effort to make the cleaning staff part of the clinical team and enhance their status.
Cleaning staff were permanently attached to various units and we almost doubled their numbers.

As well, when we were trying to figure out why our initial efforts weren't working, we noticed all the old fabric furniture, stretchers and mattresses with cracks. We threw out the fabric furniture and put big warning signs on the dumpsters: 'Do not take home. Contaminated.' We replaced fabric furniture with vinyl, which is easier to clean. We cleaned old curtains or replaced them. We did a hospital-wide decluttering and sent off truckloads of stuff. We stopped using stainless steel bedpans and brought in Vernacare, which we had already budgeted for. You use a fibre bed pan, take it to a machine like small washing machine, close it up, and macerate it.

One problem is that universities aren't training enough infection prevention and control specialists - finding someone can be difficult. It's extremely important that infection control specialists be knowledgeable but also be able to teach and lead — to bring his/her knowledge to get implemented on the ward. If that is missing, you don't get traction.

I had a medical student put together a synopsis of what we did, with some costs attached, but no, we didn't do a costeffectiveness calculation. We probably don't have the staff or time in finance to do that.

We justified what we did on the basis of shortened lengths of stay, fewer complications, less need for private rooms and saving down the road. I think we have accomplished all of that. - Ann Silversides, CMAJ

DOI:10.1503/cmaj.109-3057 